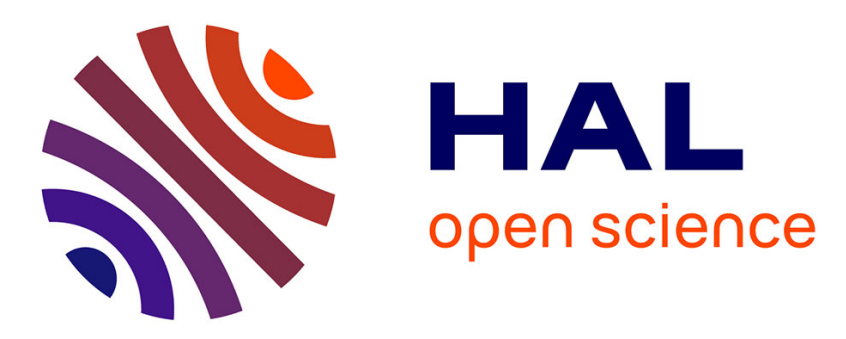

\title{
Synthèses comparées d'AlSb par recuit laser et décharge capacitive
}

\author{
L. Baufay, R. Andrew, A. Pigeolet, L.D. Laude
}

\section{To cite this version:}

L. Baufay, R. Andrew, A. Pigeolet, L.D. Laude. Synthèses comparées d'AlSb par recuit laser et décharge capacitive. Revue de Physique Appliquée, 1983, 18 (4), pp.207-212. 10.1051/rphysap:01983001804020700 . jpa-00245085

\section{HAL Id: jpa-00245085 https://hal.science/jpa-00245085}

Submitted on 1 Jan 1983

HAL is a multi-disciplinary open access archive for the deposit and dissemination of scientific research documents, whether they are published or not. The documents may come from teaching and research institutions in France or abroad, or from public or private research centers.
L'archive ouverte pluridisciplinaire HAL, est destinée au dépôt et à la diffusion de documents scientifiques de niveau recherche, publiés ou non, émanant des établissements d'enseignement et de recherche français ou étrangers, des laboratoires publics ou privés. 
Classification

Physics Abstracts

$61.80-81.10-81.20-52.50-61.50$

\title{
Synthèses comparées d'AISb par recuit laser et décharge capacitive
}

\author{
L. Baufay, R. Andrew, A. Pigeolet et L. D. Laude. \\ Université de l'Etat, 7000 Mons, Belgique
}

(Reçu le 9 août 1982, révisé le 17 janvier-1983, accepté le 19 janvier 1983)

\begin{abstract}
Résumé. - La synthèse de film mince d'AlSb est réalisée à partir de films sandwiches $\mathrm{Al} / \mathrm{Sb}$ soumis soit à un rayonnement laser, soit à une décharge capacitive. La comparaison entre ces deux techniques permet une meilleure compréhension de la nature de la transformation. L'énergie de seuil nécessaire pour que la transformation soit complète (dans le cas de films multicouches dont l'épaisseur totale est de l'ordre de $1000 \AA ̊$ ) est mesurée en fonction de la durée de la pulsation, sur une gamme s'étendant de $10^{-7}$ à $10^{-1} \mathrm{~s}$. La température induite par ces pulsations d'énergie est ensuite calculée être d'au moins $900 \mathrm{~K}$, soit approximativement les points de fusion des composants.
\end{abstract}

\begin{abstract}
The preparation of AlSb films by pulsed laser is compared to a capacity discharge annealing of $\mathrm{Al} / \mathrm{Sb}$ sandwiches in order to shed light upon the nature of the transformation. Using $1000 \AA$ thick multilayer films supported by glass substrates, we investigate the energy threshold for complete transformation as a function of (laser or capacity discharge) pulse duration from $10^{-7}$ to $10^{-1} \mathrm{~s}$. We then calculated the temperature raise directly induced by the energy pulse to be, in the two regimes, at least $900 \mathrm{~K}$, which is approximately the melting points of the metallic components.
\end{abstract}

1. Introduction. - Au cours des dernières années, les lasers se sont révélés être de puissants outils pour la recristallisation de monocristaux amorphisés lors d'implantation ionique ou même pour la cristallisation de semiconducteurs élémentaires [1]. De tels recuits ont aussi été réalisés en utilisant des faisceaux électroniques [1] ou un rayonnement microonde [2]. La nature et la modélisation de telles transformations ont suscité et suscitent encore maintes discussions : sont-elles d'origine thermique ou pas ? Une comparaison entre produits résultant soit de l'irradiation par une pulsation laser soit d'un traitement par un pulse purement thermique (ayant les mêmes caractéristiques de durée et de puissance) permettrait sans doute de mieux appréhender le problème.

Cette comparaison, nous avons essayé de la réaliser dans un autre domaine du traitement des matériaux par le laser, en vue de mieux comprendre les réactions que celui-ci induit. En effet, nous avons précédemment rapporté la possibilité de synthétiser des composés semiconducteurs $\mathrm{AB}$ en exposant à un rayonnement laser un film multicouche constitué par les composants A et B [3-5]. Il a ainsi été possible d'obtenir des films d'AlSb, CdTe, CdSe, ...; leur caractérisation a déjà été rapportée $[3,5,10]$ et semble indiquer qu'ils sont de très bonne qualité. Quant aux mécanismes de ces réactions de synthèse, ils furent étudiés sur des films dits libres (c. à d. détachés de leur substrat de sel et transférés sur une grille de microscope électronique) [6-8]. Un modèle purement thermique fut montré rendre compte de la transformation induite par le recuit laser. La chaleur latente de formation du composé y joue un rôle essentiel, contrairement à ce qui se passe pour un recuit classique dans un four. Dans le premier cas, la réaction est isoénergétique, dans l'autre cas, elle est isothermique.

Néanmoins, ce modèle fut établi pour des films dits "libres ", et il nous semblait intéressant d'étudier le cas de films supportés par un substrat isolant, celui-ci pouvant notamment jouer le rôle de puits thermique. En vue de répondre aux éventuels arguments en faveur d'effets non thermiques (notamment dans le cas de pulsation laser de $1 \mu \mathrm{s}$ ), la pulsation laser est ici simulée, tant du point de vue énergie que durée, par la décharge d'une capacité à travers le film. Dans cet article, la comparaison entre les deux types de traitement, irradiation laser en régime pulsé et décharge capacitive, permet d'étendre le modèle, précédemment proposé dans le cas de films libres, au cas des films supportés. Nous avons, ici, choisi de traiter, à titre exemplatif, de la synthèse d'AlSb.

Notons (sans pour autant assimiler synthèse de composés semiconducteurs à partir de films métalliques et cristallisation de $\mathrm{Si}$ ou $\mathrm{Ge}$ amorphe) que la décharge capacitive est aisément réalisable dans ce 
travail en raison de la faible résistivité électrique de nos échantillons (métalliques) avant irradiation, alors qu'elle serait plus difficile à mettre en œuvre pour des matériaux tels que le silicium ou le germanium qui présentent une résistivité électrique élevée. En effet, considérons à titre d'exemple un échantillon dont les caractéristiques géométriques sont les suivantes : longueur : 3,6 mm; largeur : $1 \mathrm{~mm}$ et épaisseur : $1000 \AA$. Si on désire distribuer par décharge capacitive, dans ce film, une énergie de $100 \mathrm{~mJ} / \mathrm{cm}^{2}$ (l'énergie est normalisée à la surface du film) dans un temps de $10^{-4} \mathrm{~s}$, on peut très facilement estimer que suivant la résistivité électrique du film, $10^{-6}$ ou $10^{3} \Omega . \mathrm{cm}$ (respectivement de type métallique ou semiconducteur), il faudra respectivement une capacité de $10 \mathrm{ou}$ $10^{-6} \mu \mathrm{F}$ chargée à une tension initiale de quelques volts ou dizaine de kilovolts. Un montage expérimental simple se prête donc mieux à une décharge capacitive à travers un film métallique.

2. Dispositifs expérimentaux. -2.1 PréParation DES FILMS. - Utilisant un canon à électrons, à creusets multiples, des couches d'Al et de Sb sont successivement et alternativement condensées sur un substrat en verre poli, initialement nettoyé par bombardement ionique. La pression initiale dans l'enceinte de préparation est d'environ $10^{-7}$ à $10^{-6}$ torr. La mesure des épaisseurs se fait par la méthode du quartz vibrant.

L'épaisseur et le nombre de chacune des couches élémentaires ( $\mathrm{Al}$ ou $\mathrm{Sb}$ ) est un paramètre ajusté de façon à obtenir, avec une incertitude inférieure à $2 \%$, un nombre égal d'atomes d'aluminium et d'antimoine sur la totalité de l'épaisseur du film, qui est ici d'environ $1000 \AA$.

$\mathrm{Au}$ cours de la même séquence d'évaporation deux types d'échantillons sont préparés et destinés respectivement à être recuit soit par laser, soit par décharge capacitive :

i) des échantillons de grande surface $\left(26 \times 76 \mathrm{~mm}^{2}\right)$ constitués soit d'une couche d'Al et d'une couche de $\mathrm{Sb}$, soit de 8 couches d'Al et de 7 couches de $\mathrm{Sb}$;

ii) 44 échantillons, dont les dimensions caractéristiques sont $3,6 \times 1,0 \mathrm{~mm}^{2}$, à l'extrémité desquels des contacts d'aluminium épais de $1 \mu$ sont par la suite déposés in situ. Ces films sont constitués soit d'une couche de chacun des constituants, soit de 3 couches d'Al et 2 de $\mathrm{Sb}$. Leur résistance électrique initiale est respectivement d'environ 5 ou $15 \Omega$.

Ces films sont protégés contre toute dégradation à l'air par une couche de $\mathrm{SiO}$, épaisse de $\sim 250 \AA$, évaporée par-dessus le film multicouche métallique dans la même enceinte à vide.

2.2 SYNTHÈSE DU COMPOSÉ SEMICONDUCTEUR AlSb. Ces échantillons métalliques sont recuits à l'air, à la température ambiante, soit par irradiation laser, soit par décharge capacitive dans le but d'obtenir le composé semiconducteur AlSb. L'identification de celui-ci a été effectuée à l'aide de différentes techniques (absorption optique, transport électrique et diffraction électronique) et publiée ailleurs [3]. Notons néanmoins que la présence d'AlSb est aisément détectée par microscopie optique. En effet, aux épaisseurs utilisées, le sandwich métallique $\mathrm{Al} / \mathrm{Sb}$ est opaque, tandis que l'antimoniure d'aluminium est fortement transparent dans la partie "orange » du spectre visible. Cette particularité a été utilisée dans ce travail pour juger de l'apparition, ou pas, d'AlSb.

2.2.1 Irradiation laser. - Deux types de faisceau laser sont ici utilisés pour irradier et transformer les films métalliques :

a) Le faisceau d'un laser à colorant pompé par une lampe flash. La pulsation qu'il délivre a une énergie photonique de $2 \mathrm{eV}$ et une durée de $\sim 1,8 \mu \mathrm{s}$. En posant $\bar{t}=t / t_{0}$, paramètre de temps sans dimension, où $t$ est le paramètre réel de temps et $t_{0}$ une constante égale à $7,5 \times 10^{-7} \mathrm{~s}$, la distribution énergétique de cette pulsation varie au cours du temps approximativement selon

$$
Q(\bar{t})=Q_{0} \bar{t} \exp \left(-\bar{t}^{2}\right),
$$

où $Q(\bar{t})$ est exprimé en $\mathrm{W} / \mathrm{cm}^{2}$, et $Q_{0}=2 E / t_{0}$ (E est l'énergie totale délivrée au cours de la pulsation, exprimée en $\mathrm{J} / \mathrm{cm}^{2}$ ).

b) Le faisceau d'un laser ionique à krypton (toutes les lignes rouges sont ici utilisées et l'énergie photonique est donc d'environ $1,9 \mathrm{eV}$ ) qui, jumelé avec un obturateur (une fente mobile), délivre des pulsations dont la durée est réglable de $\sim 10^{-4}$ à $\sim 10^{-1} \mathrm{~s}$. Le profil temporel de celles-ci peut être approché par une pulsation trapézoïdale :

$$
\begin{aligned}
& Q(\bar{t})=Q_{0}[5 \bar{t} H(\bar{t})+(1-5 \bar{t}) H(\bar{t}-0,2)+ \\
& +(4-5 \bar{t}) H(\bar{t}-0,8)+5(\bar{t}-1) H(\bar{t}-1)],
\end{aligned}
$$

où

$Q(\bar{t})$ est exprimé en $\mathrm{W} / \mathrm{cm}^{2}$;

$\bar{t}=t / t_{0}$ est le paramètre temps non dimensionné ( $t$ est le paramètre réel du temps et $t_{0}$ est ici la durée réelle de la pulsation);

$E=Q_{0} t_{0}$ est l'énergie totale délivrée au cours de la pulsation.

La fonction de Heaviside est définie par :

$$
\begin{aligned}
& H(x-a)=0 \text { pour } x<a \\
& H(x-a)=1 \text { pour } x>a .
\end{aligned}
$$

Pour différentes durées de pulsation laser, on détermine l'énergie minimum $E_{\mathrm{L}}$, corrigée pour la réflection [9], nécessaire pour transformer le film (remarque : pour une énergie inférieure à $E_{\mathrm{L}}$, le film reste métallique et opaque par microscopie optique; pour une énergie supérieure, l'apparition d'AlSb est détectée par transparence; voir $\S 2.2$ ). Dans ces expériences, nous ne tenons pas compte de la légère différence d'énergie photonique des deux types de faisceaux 
laser (d'autres expériences mettant en œuvre un laser ionique à argon indiquaient qu'il n'y a aucune dépendance substantielle en fonction de la longueur d'onde émise par le laser [6]). La figure 1 représente cette énergie de seuil $E_{\mathrm{L}}$ en fonction de la durée de la pulsation. Cette courbe est essentiellement différente de celle obtenue précédemment dans le cas de films dits libres [6-8], où deux régimes distincts étaient mis en évidence : l'un à énergie constante, l'autre à puissance constante; le passage de l'un à l'autre intervenait alors pour une durée de pulsation donnée, fonction de la géométrie de la grille de microscope sur laquelle était monté le film. Ici, pour des films supportés, un seul régime est à noter : l'énergie est proportionnelle à la racine carrée de la durée de la pulsation.

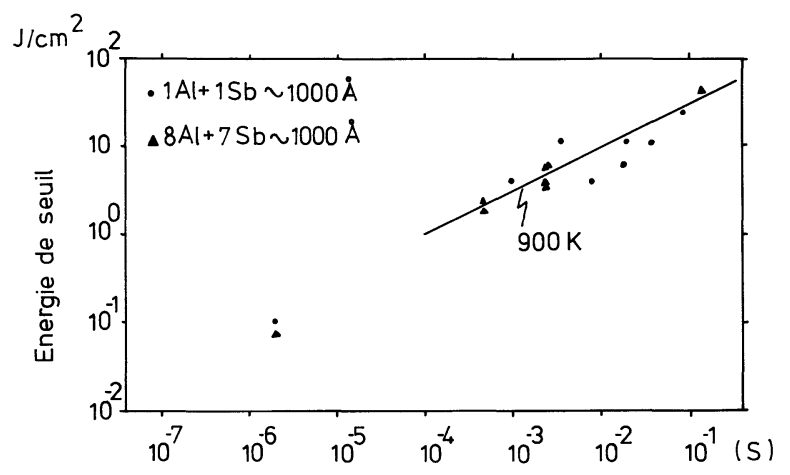

Durée du pulse laser

Fig. 1. - Energie de seuil du pulse laser nécessaire à la transformation, en fonction de la durée de la pulsation. La courbe isotherme est également représentée.

[Laser pulse energy necessary as a function of pulse duration for transformation under threshold conditions. The isothermal curve is also drawn.]

2.2.2 Décharge capacitive. - Les échantillons sont introduits dans un circuit résistance-condensateur (Fig. 2). Une capacité $(C)$ dont la tension de charge initiale est $V(0)$ se décharge dans une résistance $\left(R+R_{\mathrm{f}}\right)$ où $R$ est la résistance électrique de l'échantillon et $R_{\mathrm{f}} \leqslant 0,1 \Omega$ est une résistance constante permettant de mesurer le courant de décharge $I(t)$. La

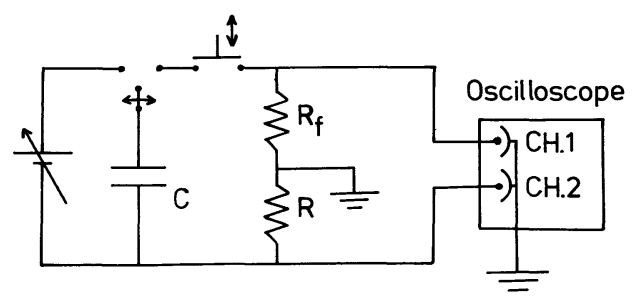

Fig. 2. - Schéma du circuit capacité-résistance utilisé pour le recuit par décharge capacitive.

[Resistance-capacitance circuit used in the capacity discharge annealing.] variation de la tension $V(t)$ est mesurée aux bornes de l'échantillon.

Il a été noté dans ce travail que pour un temps caractéristique de décharge, c'est-à-dire pour toutes valeurs de $R C / 2$, il existe une valeur critique de la tension initialement appliquée à l'échantillon $V_{\text {crit }}(0)$.

En effet, quand $V(t=0)<V_{\text {crit }}(0)$, la décharge classique d'une capacité $C$ à travers une résistance constante $\left(R+R_{\mathrm{f}}\right) \cong R$ est observée :

$$
Q(t)=V(t) I(t)=\frac{V^{2}(t=0)}{R} \exp \left(-\frac{2 t}{R C}\right) .
$$

Dans ce cas, la résistivité du film n'est pas modifiée et le film reste opaque par transparence et donc métallique.

Par contre, si $V(t=0)>V_{\text {crit }}(0)$, il apparaît un temps critique $\theta$ fonction de $V(t=0)$. Pour $t<\theta$, la décharge classique d'une capacité $C$ à travers une résistance constante $R$ est noté ; pour $t=\theta$, cette décharge est brutalement interrompue, ensuite, la tension aux bornes de l'échantillon reste constante et le courant de décharge reste nul. Ce temps $\theta$ tend vers 0 si on augmente la tension $V(t=0)$, il tend vers l'infini si on diminue $V(t=0)$ et fait tendre la tension initiale vers $V_{\text {crit }}(0)$ (voir Fig. 3). Cet arrêt de la décharge est associé à la formation du composé AlSb dont la résistivité est quelque dix ordres de grandeur plus élevée que celle du film métallique initial.

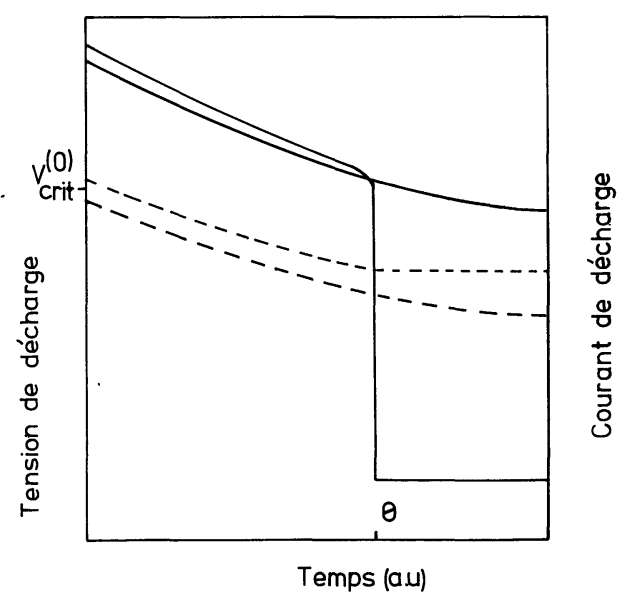

Fig. 3. - Courant (-) et tension (- - -) de décharge typique observés lorsque la tension initiale aux bornes de l'échantillon est soit plus grande, soit plus petite que la valeur critique $V_{\text {crit }}(0)$.

[Current (-) and tension (- - ) discharges typically observed when the initial tension is either greater or less than the critical value $V_{\text {crit }}(0)$.]

Utilisant des films de même résistance $R$, et pour une même capacité $C$, nous avons répété l'expérience en faisant varier $V(t=0)$. En ajustant cette valeur de tension initiale autour de la valeur critique, il est possible de déterminer par interpolation linéaire (à moins de $5 \%$ près) la valeur de $V_{\text {crit }}(0)$ qui est un seuil 
à franchir pour obtenir la transformation métalsemiconducteur. On peut dès lors calculer l'énergie minimum $C V_{\text {crit }}^{2}(0) / 2$ dissipée dans l'échantillon et nécessaire à la transformation.

Comme indiqué par la relation (3), le temps caractéristique d'une décharge capacitive, en terme de puissance injectée, est $R C / 2$. La figure 4 représente

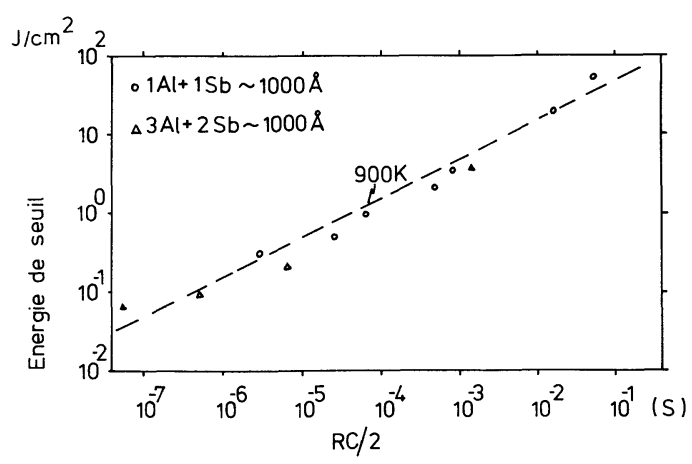

Fig. 4. - Energie de seuil nécessaire à la transformation, mesurée lors des expériences de décharge capacitive, en fonction de $R C / 2$. La courbe isotherme est également représentée.

[Threshold energy as a function of $R C / 2$. The isothermal curve is also drawn.]

$E=C V_{\text {crit }}^{2}(0) / 2$ normalisée pour la surface de l'échantillon, en fonction de $R C / 2$. Cette énergie de seuil varie suivant la racine carrée de $R C / 2$.

Remarquons enfin que la formation d'AlSb induite par ce type de recuit a toujours été, dans nos expériences, accompagnée par la perforation du film, comme montré à la figure 5 .

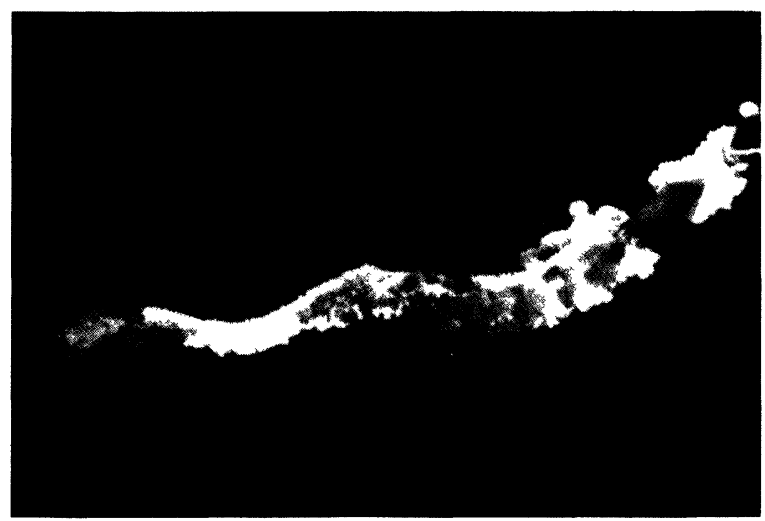

$50 \mu \mathrm{m}$

Fig. 5. - Vue microscopique d'échantillon typique recuit par décharge capacitive. La partie noire est métallique $(\mathrm{Al}+\mathrm{Sb})$, la partie jaune est constituée d'AlSb, la zone blanche correspond au trou formé lors du claquage.

[Microscopic view of a typical sample which have been annealed by capacity discharge. The black zone is metallic $(\mathrm{Al}+\mathrm{Sb})$, the yellow region correspond to $\mathrm{AlSb}$, and the hole (white part) is formed during the breakdown process.]
3. Discussion. - Le composé semiconducteur a pu être synthétisé à partir des composants métalliques et ce par les deux techniques de recuit envisagées ici. Dans chaque cas, nous avons déterminé les énergies de seuil nécessaires à la transformation. Que veut dire énergie de seuil et quelles sont les conclusions que l'on peut tirer de ces mesures ?

Pour des valeurs de l'énergie de pulse supérieure à la valeur de seuil, la transformation métal-semiconducteur provoque une variation abrupte et importante de la résistivité électrique et de l'absorption optique. La distribution temporelle de puissance réellement absorbée par le film est affectée par cette transformation et n'est donc plus représentée par les équations (1), (2) ou (3) donnant suivant le type de pulse envisagé la distribution temporelle de l'excitation. (Cette remarque est d'ailleurs illustrée par la Fig. 3.) Ceci conduit à des résultats expérimentaux différents suivant qu'il s'agit d'une excitation par décharge capacitive (DCa) ou par irradiation laser (IL). En effet, il a été noté dans le premier cas que le film est partiellement détruit (Fig. 5), alors qu'aucune dégradation n'apparaît lors du recuit par faisceau laser. Ce comportement différent résulte de la formation du composé semiconducteur. Dans les expériences $\mathrm{DCa}$, cette synthèse, lorsqu'elle est initialisée en un site du film, y provoque une augmentation locale importante de la résistance électrique et force la puissance électrique à être dissipée dans les zones métalliques avoisinant la région où est apparu AlSb. (En effet, lors de l'apparition d'AlSb en un site, la tension aux bornes de l'échantillon n'est pas nécessairement nulle.) Ce phénomène, et en même temps la croissance d'AlSb, s'accélèrent en un processus avalanche, et résultent en la formation d'une fine tranche d'AlSb soumise à un champ électrique important. Un claquage intervient alors à la fin du processus. Par ailleurs, la formation d'AlSb par rayonnement laser est auto-régulée en ce sens qu'elle réduit fortement l'absorption optique et donc l'énergie dissipée localement (dès la formation du composé pendant la durée de la pulsation, l'absorption des photons est fortement réduite).

Dans le cas de tir moins énergétique (sous la valeur de seuil) aucune transformation n'est notée : le film reste métallique, sa résistance électrique et son absorption optique demeurent donc inchangées au cours du tir (DCa ou IL). Les expressions (1), (2) et (3) donnant la distribution temporelle de puissance excitatrice pour chaque type de pulse représentent donc aussi la distribution de puissance réellement absorbée par le film. Ceci est vrai jusqu'au seuil de la transformation et nous permet de comparer les deux types de tir (DCa ou IL) en ne considérant que les valeurs de l'énergie de seuil.

La comparaison des figures 1 et 4 où sont reportées les énergies minima nécessaires à la transformation, suggère que le processus présidant à la formation d'AlSb est identique pour les deux types de recuit. 
Ces deux courbes montrent en effet une même dépendance de l'énergie de seuil nécessaire à la transformation en fonction du temps caractéristique de la pulsation d'énergie. Les valeurs absolues y sont, de plus, fort semblables.

Tout comme dans le cas de la décharge capacitive, la transformation sous rayonnement laser est d'origine thermique.

Le calcul de la température induite par ces pulsations d'énergie peut être réalisé aisément à l'aide d'hypothèses raisonnables :

1) la capacité thermique du film est négligeable comparée à celle du substrat (cette hypothèse est d'autant mieux vérifiée que la pulsation est courte);

2) le contact film/substrat est parfait au point de vue échange de chaleur;

3) la température est uniforme sur l'épaisseur du film (remarquons que les valeurs de diffusivité thermique $\kappa$ dans les matériaux massiques sont respectivement de 0,86 et $0,15 \mathrm{~cm}^{2} / \mathrm{s}$ pour l'aluminium et l'antimoine; la longueur de diffusion de chaleur $\sqrt{\kappa t}$ pour des temps de $\sim 10^{-7} \mathrm{~s}$ est ainsi au moins 10 fois l'épaisseur du film;

4) le substrat est semi-infini.

Avec de telles hypothèses, le comportement thermique du film est simplement régi par lè substrat. La température du film est celle de la surface du substrat. Le problème est alors réduit au cas unidimensionnel du calcul de température $T(z=0, t)$ dans un solide semi-infini ayant une conductivité thermique
$K=9 \times 10^{-3} \mathrm{~W} / \mathrm{cm} . \mathrm{K}$ et une diffusivite thermique $\kappa=3,5 \times 10^{-3} \mathrm{~cm}^{2} / \mathrm{s}$, soumis à un flux de chaleur $Q(t)$ en $z=0, Q(t)$ est alors défini par les équations (1), (2) ou (3) suivant le type de recuit envisagé. Donc,

$$
\frac{\partial^{2} T}{\partial z^{2}}-\frac{1}{\kappa} \frac{\partial T}{\partial t}=0,
$$

doit être résolu avec les conditions limites

$$
\begin{gathered}
\left.\frac{\partial T}{\partial z}\right|_{z=0}=-\frac{Q(t)}{K} \\
\lim _{z \rightarrow \infty} T(z, t)=T_{\mathrm{i}}=300 \mathrm{~K}, \\
T(z, t=0)=T_{\mathrm{i}}=300 \mathrm{~K} .
\end{gathered}
$$

( $T$ est le paramètre température, $z$ et $t$ sont respectivement les variables d'espace et de temps, $T_{i}$ est la température initiale du système.)

Ce calcul est simplifié par un choix judicieux de paramètres non dimensionnés :

$$
\bar{T}=\left(T^{-}-T_{\mathrm{i}}\right) / T_{0}, \quad \bar{z}=z / z_{0} \quad \text { et } \quad \bar{t}=t / t_{0},
$$

en posant

$$
z_{0}={\sqrt{\kappa t_{0}}}_{0}, \quad T_{0}=z_{0} Q_{0} / K
$$

où $t_{0}$ et $Q_{0}$ ont été définis précédemment pour le cas de l'irradiation laser, alors que pour la décharge capacitive, on choisit de préférence :

$$
t_{0}=R C / 2 \quad \text { et } \quad Q_{0}=\frac{V_{\text {crit }}^{2}(0)}{R}=\frac{C V_{\text {crit }}^{2}(0)}{2} \frac{1}{t_{0}} .
$$

Utilisant les transformées de Laplace et le théorème de Duhamel, on peut montrer que pour :

- la décharge capacitive :

$$
\bar{T}(0, \bar{t})=\frac{2}{\sqrt{\pi}} \mathrm{e}^{-\bar{t}} \int_{0}^{\sqrt{\bar{t}}} \exp \left(\lambda^{2}\right) \mathrm{d} \lambda,
$$

- l'irradiation par le laser à colorant :

$$
\bar{T}(0, \bar{t})=\frac{4}{\sqrt{\pi}} \int_{0}^{\bar{t}} \sqrt{\bar{t}-\theta} \exp \left(-\theta^{2}\right)\left(1-2 \theta^{2}\right) \mathrm{d} \theta,
$$

- l'irradiation par le laser à krypton :

$\bar{T}(0, \bar{t})=\frac{20}{3 \sqrt{\pi}}\left\{\bar{t}^{3 / 2} H(\bar{t})-(\bar{t}-0,2)^{3 / 2} H(\bar{t}-0,2)-(\bar{t}-0,8)^{3 / 2} H(\bar{t}-0,8)+(\bar{t}-1)^{3 / 2} H(\bar{t}-1)\right\}$.

Sont reprises à la figure 6 les différentes pulsations de chaleur induites par les pulsations d'énergie; ainsi que les valeurs maxima obtenues pour la température $\bar{T}$.

Remarquons qu'une isotherme est définie par :

$T=\bar{T} T_{0}+T_{\mathrm{i}}=$ Cte $\sim T_{0} \sim Q_{0}{\sqrt{\kappa t_{0}}}_{2} \sim{ } / \sqrt{t}_{0}$, où $E$ est l'énergie dissipée dans l'échantillon et $t_{0}$ une durée caractéristique de pulsation.

Les figures 1 et 4 (où l'énergie de seuil pour la transformation est proportionnelle à la racine carrée du temps caractéristique de la pulsation) montrent donc que le processus est isotherme dans la gamme de temps 


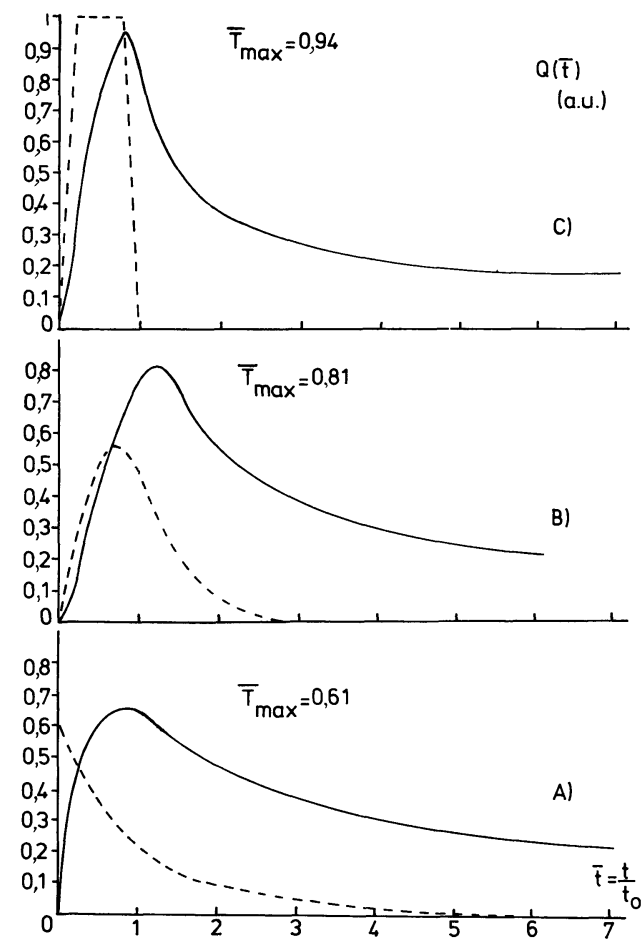

Fig. 6. - Courbes représentant la distribution en puissance des pulses produits par : A) décharge capacitive; B) le dye laser; C) par le laser ionique (- - ). Est également représentée l'évolution de la température calculée (grandeur ici sans dimension) induite par ces différents pulses (-).

[These curves represent the power distribution as a function of the time for pulses delivered by : A) discharge capacity; B) the dye laser; C) the krypton ion laser. The temperature (dimensionless parameter) variation induced by these pulses are calculated and represented. (Input power distribution : - - - temperature distribution : - - )] étudiée. Cette température induite est calculée selon les figures 1,4 et 6 être d'environ $900 \mathrm{~K}$.

En conclusion, quels que soient le type de tir et la durée de celui-ci $\left(10^{-7}\right.$ à $\left.10^{-1} \mathrm{~s}\right)$, ceux-ci doivent induire une température de $900 \mathrm{~K}$ afin d'obtenir la synthèse d'AlSb. Cette température est indépendante du nombre d'interfaces $\mathrm{Al} / \mathrm{Sb}$ présentes dans le film initial ; ceci exclut une croissance classique d'AlSb en phase solide à partir d'une interface $\mathrm{Al} / \mathrm{Sb}$ et par diffusion d'un (ou des deux) constituant(s) à travers la tranche d'AlSb croissante, ce qui impliquerait alors une dépendance de l'épaisseur d'AlSb formée en fonction de la température et du temps de réaction [11]. Ceci conduit à confirmer la fusion des constituants.

Dès lors le modèle établi pour les films libres [6-8] est toujours valable, la seule différence entre ce type de film et celui traité ici (films supportés) réside dans le type de pertes thermiques. Dans le présent travail, c'est le substrat qui gouverne la température du film.

Reprenant le précédent modèle, on peut dire qu'à $900 \mathrm{~K}$, ce qui correspond aux températures de fusion des constituants, intervient un mélange rapide des phases liquides $\mathrm{Al}$ et $\mathrm{Sb}$ qui résulte en la formation d'AlSb, dans la libération de l'énergie de formation de ce composé et donc en un accroissement rapide de la température qui rend la réaction autonome et plus rapide.

Remerciements. - M. Wautelet doit être remercié pour les discussions auxquelles il a participé dans le cadre de ce travail financé par le projet énergie du Ministère Belge de la Politique Scientifique (contrat $\mathrm{E} / \mathrm{XII} / 4)$.

\section{Bibliographie}

[1] Voir par exemple les proceedings de MRS, Réunion annuelle, Laser and Electron Beam Solid Interactions and Materials Processing vol. 1 (ed. North Holland) 1980.

[2] Chenevier, P., Cohen, J. and Kamarinos, G., $C$. $R$. Hebd. Séan. Acad. Sci. 294 (1982) 237-240.

[3] Andrew, R., Ledezma, M., Lovato, M., WauteLET, M. and LAUde, L. D., Appl. Phys. Lett. 35 (1979) 418.

[4] Andrew, R., Baufay, L., Laude, L. D., Lovato, M. and Wautelet, M., J. Physique colloq. 41 (1980) C4-71.

[5] Baufay, L., Dispa, D., Pigeolet, A. and Laude, L. D., J. Cryst. Growth 59 (1982) 143-147.

[6] Andrew, R., Baufay, L., Pigeolet, A. and Laude, L. D., J. Appl. Phys. 53 (7) (1982) 4862 4865.

[7] Andrew, R., Baufay, L., Pigeolet, A. and LAUde, L. D., Proceedings of MRS Annual
Meeting 1981, Laser and Electron-Beam Interactions with Solids, Vol. 4 ; eds B. R. Appleton and G. K. Celler (North Holland) 1982 p. 719-724.

[8] Baufay, L., Andrew, R., Pigeolet, A. and LAUde, L. D., Thin Solid Films 90 (1981) 69-74.

[9] La réflectivité des échantillons métalliques est déterminée avant irradiation et est ici supposée constante durant le pulse laser. Elle est mesurée en utilisant le laser ionique à krypton, à une puissance extrêmement faible $\left(15 \mathrm{~W} / \mathrm{cm}^{2}\right)$ afin d'éviter une transformation de l'échantillon lors de la mesure.

[10] Baufay, L., Pigeolet, A., Andrew, R. and LAUDE, L. D., submitted for publication in the Proceedings of the MRS symposium of LaserSolid Interactions and Transient Thermal Processing of Materials, Boston, November 1982.

[11] Treatise on Solid State Chemistry, Vol. 4, Reactivity of Solids, ed. by N. B. Hannay (Plenum Press) 1976. 